\title{
Un modelo fallido de desarrollo: la experiencia argentina (1989-2001)
}

\author{
Ernesto R. GANTMAN \\ Universidad de Buenos Aires y Universidad de Belgrano \\ ergant@sinectis.com.ar
}

Recibido: 04-01-2012

Aceptado: $16-05-2012$

\begin{abstract}
RESUMEN
El presente trabajo analiza el modelo de desarrollo emprendido por la República Argentina bajo la administración Menem, cuando se llevaron a cabo profundas transformaciones estructurales destinadas a ampliar la esfera del mercado y reducir la presencia del Estado en la economía. Si bien los resultados fueron muy negativos en materia social, particularmente en términos de aumento del desempleo y la desigualdad social, la economía tuvo un crecimiento importante durante el período. Sin embargo, la masiva acumulación de deuda externa determinó que el modelo de desarrollo adoptado colapsara en 2001.
\end{abstract}

Palabras claves: neoliberalismo, Argentina, desarrollo económico

\section{A failed model of development: The Argentine experience (1989-2001)}

\begin{abstract}
This paper analyses the model of development adopted by the Menem Administration in Argentina, when a deep structural adjustment was carried out in order to reduce the State's burden upon the economy While the results involved important negative social effects, particularly in terms of rising unemployment and social inequality, the economy experimented high growth rates during the period. However, the massive accumulation of external indebtedness precipitated the model's collapse in 2001.
\end{abstract}

Keywords: neoliberalism, Argentina, economic development

\section{REFERENCIA NORMALIZADA}

Gantman, E.R. (2012). Un modelo fallido de desarrollo: la experiencia argentina (1989-2001). Cuadernos de Relaciones Laborales Vol. 30, núm. 2, p. 327-354.

SUMARIO: Introducción. 1. Ajuste estructural: reducir el estado para agrandar la economía. 2. Cambios en el paisaje corporativo: la extranjerización de la economía en los noventa. 3. Las consecuencias sociales del ajuste: desempleo, desigualdad y criminalidad. 4. El colapso y su salida. 5. Discusión y conclusiones. 6. Referencias bibliográficas. 


\section{Introducción}

En los años noventa, la mayor parte de los países de América Latina llevaron a cabo profundas reformas estructurales, obedeciendo los dictados del denominado "consenso de Washington". Los objetivos centrales de ese diagnóstico, asociado a la ideología neoliberal, apuntaban a reducir el Estado, percibido como un lastre excesivo que ahogaba la libre empresa y la iniciativa privada, y a abrir la economía al capital extranjero, considerado como fuente de modernización y desarrollo. Las economías más grandes de la región comenzaron a ser catalogadas con la controvertida etiqueta de "mercados emergentes" y se convirtieron en receptores de capital extranjero, tanto productivo como especulativo, en una economía mundial en la cual el sistema capitalista adquirió la absoluta e indiscutida supremacía. En este trabajo, se analiza un caso particular, el de la República Argentina, una experiencia emblemática en materia de ajuste estructural dada la profundidad de las reformas adoptadas. Ningún país de la región llevó a cabo reformas tan ambiciosas en un lapso de tiempo tan corto. El caso argentino en los años noventa demostró que el país alcanzó niveles razonables de crecimiento económico, mediante el establecimiento de un modelo particular de desarrollo que algunos autores (Conesa, 2000) han denominado crecimiento liderado por la deuda (debt-led growth), en el cual, sin embargo, los logros en materia de modernización y estabilidad contrastan con consecuencias indeseables en lo social. A la postre, la propia lógica del modelo causó su inevitable estallido, sumiendo al país en una crisis económica sin precedentes. La recuperación, no obstante, llegó en unos años, con un gobierno que emprendió políticas que otorgaban un rol más activo al Estado, pero al amparo de un contexto internacional muy favorable.

En la próxima sección, describo las características fundamentales de las reformas adoptadas durante los años noventa. Luego, examino las consecuencias de las reformas en la estructura productiva del país, centrando mi análisis en el proceso de "extranjerización" de la economía, cuando importantes activos de empresas locales pasaron a manos de capitales extranjeros. A continuación, analizo las consecuencias sociales de las reformas en términos de desempleo, equidad distributiva y criminalidad. La siguiente sección comenta muy brevemente, y a efectos meramente ilustrativos, cómo se recuperó el país luego de la crisis, adoptando un modelo de desarrollo en algunos aspectos antitético, pero que tampoco logró revertir los problemas sociales básicos del país. Por último, discuto las lecciones que pueden obtenerse de este caso de estudio, que si bien constituye una experiencia extrema, demuestra qué puede ocurrir cuando se produce una indiscriminada apertura a la economía global, desatendiendo otros aspectos más básicos de prudencia en materia de política económica. 


\section{Ajuste estructural: reducir el estado para agrandar la economía}

En 1983, luego de siete años de dictadura militar, un nuevo gobierno elegido a través de un proceso electoral asumió el poder en la República Argentina. La herencia de la que se hizo cargo no era envidiable: una deuda externa contraída durante el régimen anterior que amenazaba con sumir al país en la inevitable bancarrota y creciente inequidad social respecto a períodos anteriores de la historia argentina (Tedesco, 1999). Este panorama no resultaba diferente del sufrido por otros países latinoamericanos al estallar la crisis de la deuda al inicio de los ochenta (GriffithJones y Sunkel, 1987). La recreación de las instituciones y los valores democráticos, que los militares se encargaron de silenciar con un gobierno represivo que incluyó más de 30.000 desaparecidos (Feitlowick, 1999), fue una de las tareas centrales que el gobierno radical electo debió realizar en una penosa transición, cuyo frente más débil fue el de la política económica. El deterioro progresivo del modelo de acumulación de sustitución de importaciones es señalado por algunos autores como uno de los determinantes últimos de la fragilidad de la democracia en el país (O’Donnell, 1988), y los esfuerzos del gobierno radical por reactivar la alicaída economía demostraron con su fracaso el definitivo agotamiento de dicho modelo. Sucesivos planes de ajuste heterodoxo para bajar la inflación y sanear el erario público arrojaron moderado éxito inicial, pero culminaron en resultados completamente insatisfactorios. El abanico de demandas sobre un Estado en quiebra parecía encerrar el germen de la propia destrucción de la democracia: la creciente cuantía de los intereses de la deuda externa, las protestas por incrementos salariales de grupos sindicalistas afines al Partido Justicialista (expresión política del movimiento populista iniciado por Juan Domingo Perón en los años 40 y derrotado en las elecciones presidenciales de 1983), el creciente déficit fiscal y las desigualdades sociales que el gobierno militar había causado a través de una política económica excluyente fueron el telón de fondo a la transición democrática signada por una indubitable fragilidad.

El Estado, que en la tradición política latinoamericana había sido tradicionalmente considerado como el locus propio de las soluciones al malestar comunitario (Wiarda, 1985), comenzó a ser crecientemente percibido como la verdadera causa de los problemas. Ello es demostrado por el paulatino pero sostenido incremento que el discurso neoconservador adquiría en los medios de comunicación y el crecimiento del caudal electoral de los partidos de centro derecha (de 1,61\% en las elecciones de 1983 a $13,5 \%$ en 1989, considerando los votos a diputados).

En línea con las recomendaciones de los organismos multilaterales de crédito, que comenzaron a adquirir protagonismo como auditores del gobierno de turno, y el consenso creciente en torno al diagnóstico neoconservador, el gobierno radical inició en 1987 un plan económico que intentaba, por un lado, disminuir el déficit estatal a través de un congelamiento de ingresos de personal al sector público y un control efectivo sobre el gasto, y por otro, iniciar una estrategia selectiva de privatización de empresas públicas para disminuir la carga que las mismas representaban para el erario público. Este plan, no obstante, fracasó debido a la oposición del 
partido justicialista, que bloqueó la iniciativa en el congreso argumentando razones de vulneración a la soberanía nacional. Mientras tanto, el déficit público seguía en aumento y el nivel de inflación, que actuaba como una suerte de termómetro del clima económico, mostraba un lento pero constante incremento. El golpe de gracia para el gobierno radical se produjo a principios de 1989, cuando se difundieron rumores de que el país entraría próximamente en default (Scavo, 1996; Majul, 1990). Si bien el déficit fiscal no era exageradamente grande en ese momento, una enorme presión contra la divisa nacional, efectuada por grandes operadores del exterior, causó una marcada devaluación de la moneda local y una huída hacia el dólar. La alta inflación inercial se disparó en un episodio que algunos economistas califican como la primera hiperinflación argentina (Graziano, 1990). En medio de este clima social marcadamente enrarecido, tuvieron lugar las elecciones presidenciales de 1989. El candidato del partido radical centró su campaña en una reducción frontal del gasto público, mientras que su rival justicialista, Carlos Menem, basó su campaña en propuestas difusas que incluían un llamamiento a una "revolución productiva" y un incremento de salarios para los trabajadores. La victoria justicialista fue masiva, a partir de su alto predicamento en los sectores sociales de menores ingresos, al tiempo que se advertía un notable incremento de la permeabilidad ideológica del discurso neoconservador, ya que la UCD, partido que lideró una coalición de centro-derecha, acaparó el 13\% de los votos.

La burguesía nacional y el resto del establishment parecían expectantes ante la victoria de un candidato populista, cuyo discurso anacrónico ignoraba las virtudes del Estado mínimo, propugnadas y demostradas en países centrales como los Estados Unidos y el Reino Unido, y las sabias recomendaciones de los organismos multilaterales de crédito. Para el asombro de muchos de estos sectores, el presidente electo realizó lo que se conoce como la media vuelta política más espectacular que conoció la Argentina en el siglo XX (Waisman, 1990). Invitando a su gobierno a dirigentes del partido neoliberal UCD y nombrando como ministro de Economía a un funcionario de uno de los holdings económicos más poderosos del país, Carlos Menem inició un ambicioso plan de privatizaciones y apertura de la economía. ${ }^{1} \mathrm{El}$ crédito inicial que despertó esta iniciativa se diluyó rápidamente, ya que el déficit fiscal seguía creciendo. De acuerdo a Gerchunoff y Torre (1996: 743), "el viraje ideológico y político de Menem no alcanzó, sin embargo, para que los grandes grupos económicos abandonaran su estado de alerta debido a la experiencia acumulada de repetidos fracasos en estabilizar la economía". Así, Argentina vivió un nuevo episodio hiperinflacionario en 1990. Para alejar el fantasma de la inflación que espantaba a potenciales inversores del exterior, se implementó un plan económico que la atacaba directamente. Dicho plan procuraba erradicar las expectativas inflacionarias, a partir del establecimiento de la convertibilidad legal de la moneda

\footnotetext{
${ }^{1}$ Irónicamente, el plan comenzó con la aerolínea de bandera y la empresa pública de telecomunicaciones, que fueron las que habían sido elegidas por el gobierno anterior.
} 
local con el dólar estadounidense. Como la base monetaria en moneda local estaba íntegramente respaldada en reservas en dólares, se excluía la posibilidad de devaluaciones futuras. Por otra parte, la emisión monetaria sin respaldo se encontraba prohibida por la Ley de Convertibilidad (vigente a partir de abril de 1991). Consecuentemente, la promesa, ciertamente incumplida, de reducir o eliminar el gasto público cimentó las bases para restablecer la confianza y el crédito internacional.

El objetivo de la política económica del presidente Menem apuntaba directamente a obtener el visto bueno de los Estados Unidos y los organismos multilaterales de crédito. Mostrar políticas económicas serias, propias de un país capitalista, era un anzuelo para atraer inversiones y generar así crecimiento en la economía. Los partidarios de este discurso sostenían que las ventajas para la población llegarían con el tiempo, fruto del efecto derrame de la riqueza. En este contexto, había que mostrar claramente que se aceptaban las recetas económicas en boga. Por ello, se incrementó la oferta de empresas públicas a privatizar. A partir de 1992, se sumaron diversas actividades y áreas: ferrocarriles, correo, gas, energía eléctrica, provisión de agua, petróleo. La celeridad y alcance de este proceso de privatizaciones fueron notorias (Margheritis, 1999; Gerchunoff y Cánovas, 1995). Prácticamente todo lo que fue alguna parte del llamado Estado empresario pasó a manos privadas. Esto generó sustanciales ingresos de fondos para el Estado. Las empresas que adquirieron los servicios privatizados realizaron ajustes de planta, despidiendo empleados $\mathrm{y}$, en ocasiones, incrementaron el precio de sus productos. En muchos casos, las tarifas se fijaban a valores internacionales, independientemente de la estructura de costos locales, incluyendo cláusulas de aumento de precio según la variación de la inflación internacional.

Quienes propiciaban el discurso neoconservador no podían sentirse más que satisfechos. La inversión externa aumentó. La inflación disminuyó drásticamente a unos de los niveles más bajos del mundo, mientras que la tasa de crecimiento del Producto Bruto Interno per cápita mostró varios años de incremento sostenido (Tabla 1) lo cual parecía el indicador más acabado de que la receta neoconservadora funcionaba. Algunos efectos colaterales no deseados ensombrecían este panorama, como veremos más adelante, pero ni siquiera el desempleo record del 18\% impidió que el presidente Menem obtuviera su reelección en 1995. La permanencia del partido Justicialista en el poder se debió a un fenómeno que muchos analistas denominaron "voto cuota" (Echegaray, 1996). La reducción de la inflación y las privatizaciones recrearon en la economía muchas instituciones capitalistas que estaban casi ausentes desde los años cuarenta, particularmente el crédito a largo plazo. Mucha gente se endeudó en dólares para adquirir viviendas y otros bienes. El miedo a una devaluación, a manos de un presidente que no fuese Menem, hizo que muchos compradores endeudados a crédito (una parte importante de la población) apoyaran su continuidad, pese a ciertas evidencias palpables de que en lo social su política económica había generado algunas dificultades. 
Tabla 1 - Crecimiento anual del P.B.I. per capita en la Republica Argentina (1990-2009)

\begin{tabular}{|l|c|}
\hline AÑO & Tasa de crecimiento (en \%) \\
\hline 1990 & -3.78 \\
\hline 1991 & 11.10 \\
\hline 1992 & 10.41 \\
\hline 1993 & 4.49 \\
\hline 1994 & 4.44 \\
\hline 1995 & -4.11 \\
\hline 1996 & 4.18 \\
\hline 1997 & 6.75 \\
\hline 1998 & 2.58 \\
\hline 1999 & -4.51 \\
\hline 2000 & -1.87 \\
\hline 2001 & -5.38 \\
\hline 2002 & -11.74 \\
\hline 2003 & 7.84 \\
\hline 2004 & 8.04 \\
\hline 2005 & 8.16 \\
\hline 2006 & 7.43 \\
\hline 2007 & 7.59 \\
\hline 2008 & 5.71 \\
\hline 2009 & -0.13 \\
\hline
\end{tabular}

Fuente: elaboración propia en base a datos del Banco Mundial (2011)

Los defensores del discurso neoconservador argumentaban que las ventajas del ajuste tarde o temprano se extenderían a la población, a partir de un "efecto derrame" del crecimiento económico. Con el tiempo, el país adquiriría el status de nación desarrollada. Este movimiento hacia el desarrollo capitalista prometía enterrar definitivamente las prácticas de clientelismo y corrupción del Estado argentino, que asfixiaban la libre empresa y sólo otorgaban beneficios a los empresarios cuyos ingresos eran el resultado de la cuasi-renta emanada de los privilegios concedidos por las regulaciones gubernamentales. Al eliminarse el Estado empresario, se erradicarían las posibilidades de corrupción. ${ }^{2}$ Pero las reformas iban más allá de la mera retirada del estado de su rol como proveedor directo de bienes y servicios. La lógica

${ }^{2}$ No obstante, es interesante señalar que diversos rumores de corrupción en la administración Menem capturaron la atención de los medios periodísticos en los años noventa (Rodríguez, 1998; Verbitsky 1992). 
de este diagnóstico requería, además, la remoción de una multiplicidad de regulaciones burocráticas que asfixiaban la iniciativa privada e impedían, entre otras cosas, el crecimiento del comercio exterior. Así, se procedió a desregular la actividad en varios sectores y a limitar las barreras a la entrada y salida de capitales. Además, se redujeron las tarifas de importación. En suma, se intentó adoptar lo que Edelstein y Chilcote (1983) denominaron modelo difusionista del desarrollo capitalista, que supone la creación de las condiciones políticas y económicas que permitan la recepción de inversiones y valores culturales provenientes de países centrales. Esto es, naturalmente, contingente del modelo de capitalismo dominante en el centro del sistema y, en la coyuntura histórica en cuestión, el discurso dominante se basaba en la imposibilidad de oponerse a la globalización capitalista y en la necesidad de acomodarse a los dictados de la economía mundial.

Así, el discurso del gobierno pintaba sus logros como un verdadero milagro. Se hablaba de que la Argentina había entrado al Primer Mundo. La justificación del desempleo, por ejemplo, era atribuida a la globalización: una fuerza misteriosa que prometía recompensa al que la abrazara y destrucción a quien la ignorara. Los beneficios del desarrollo capitalista llegarían más tarde o más temprano. No obstante, este discurso que tenía un tono casi mágico omitía ciertos hechos básicos. En primer lugar, la llegada de inversiones a Argentina, si bien fruto de la expansión del capitalismo a escala global, era consecuencia directa de un proceso de liquidez internacional creciente. Otros países emergentes que no realizaron procesos de privatización tan profundos recibieron también importantes niveles de inversión extranjera directa. Los capitales financieros se mostraban poco racionales y proclives al "comportamiento de manada", lo que indudablemente contribuyó a la internacionalización de crisis financieras como la de México en diciembre de 1994 (efecto Tequila).

Sin embargo, es necesario puntualizar que la lógica del discurso neoconservador no se aplicó al pie de la letra en Argentina durante el gobierno peronista. El déficit público continuó siendo un problema, a pesar la inyección de fondos provenientes de las privatizaciones. A fines del 2000 alcanzó a 2,4\% del PIB, según datos de la CEPAL (2001). El déficit público se financiaba con deuda externa, ya que la emisión monetaria era una alternativa eliminada por el propio plan de convertibilidad. En definitiva, el modelo de crecimiento se basaba en el endeudamiento y no tenía una base sólida en la economía real, pese a algunos indicadores de expansión económica, particularmente vinculados al sector servicios. Más bien, todo el andamiaje de la economía reposaba en los humores volátiles de los acreedores externos y, fundamentalmente, en los vaivenes de liquidez del capitalismo global. Por otra parte, si bien el tema de la devaluación de la moneda local era un tabú entre los economistas argentinos, el miedo a la devaluación tenía bases reales, dada la sobrevaluación de la moneda. Como se aprecia en la Figura 1, el índice de 100 que corresponde a una de los primeros años del plan de convertibilidad (1993), es significativamente inferior a cualquier valor de la década anterior. 
Figura 1 - Indice de tipo de cambio real dolar/peso (1982-2007)

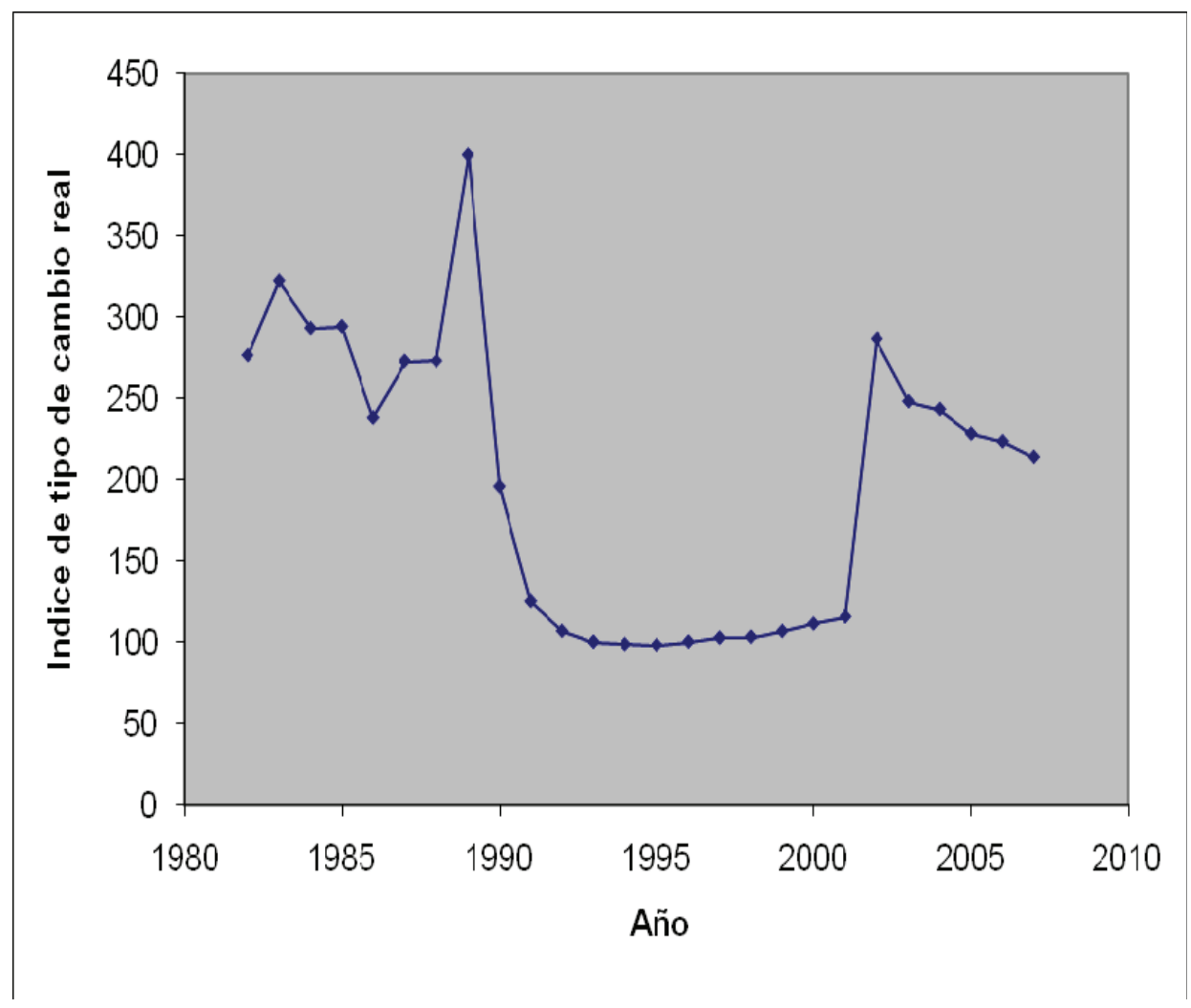

Nota: $1993=100$

Fuente: elaboración propia en base a datos de CEPAL (2011a)

Paulatinamente, la economía del país comenzó a mostrar signos de que el modelo de crecimiento liderado por el incremento de deuda externa entraba en una fase de agotamiento. El partido justicialista perdió en las elecciones presidenciales de 1999 (el propio presidente Menem se presentó como candidato luego de una reforma constitucional que, entre otras, posibilitó su nueva candidatura). Sin embargo, el candidato radical que resultó victorioso liderando una alianza de partidos de oposición tuvo que manifestar claramente en su campaña que mantendría a rajatabla la paridad cambiaria. Al asumir el gobierno, quedó claro que sus políticas económicas eran la continuidad de las políticas de Menem. Naturalmente, el gasto público heredado del gobierno anterior era alto. La tasa de interés internacional había aumentado $\mathrm{y}$, consecuentemente, los intereses de la enorme deuda externa adquirían una dimensión preocupante. Ya no quedaban activos estatales para vender. Desde la política económica, la respuesta adoptada fue un incremento de impuestos y, com- 
probada la ineficiencia del mismo para alcanzar un nivel de déficit público tolerable, una reducción de los sueldos de los empleados públicos que afectó a la casi totalidad de la dotación de personal. Como veremos más adelante, todo resultó inútil y el inevitable colapso del modelo finalmente sobrevino.

La precedente narración ha situado al lector respecto al proceso de reforma estructural de la economía argentina. Con el objeto de promover el desarrollo económico y reducir la brecha que separa al país de las economías centrales, se adoptó fielmente un conjunto de medidas propugnadas por el diagnóstico neoliberal: desregulación, apertura de la economía, eliminación de trabas al ingreso de capitales, privatización total de empresas pública. Hubo cierto nivel de crecimiento y de modernización de la economía, pero el desarrollo económico no llegó. Antes bien, el modelo colapsó sumiendo al país en una crisis sin precedentes. Para los capitales provenientes de países centrales, sin embargo, la política económica argentina de los años noventa fue beneficiosa. No sólo incrementaron sus negocios con el país, sino que obtuvieron interesantes beneficios por ello. Para entender mejor, estos desarrollos presentaré seguidamente las transformaciones ocurridas en el mundo empresarial.

\section{Cambios en el paisaje corporativo: la extranjerización de la economía en los noventa}

Según la teoría de la ecología organizacional, el cambio en la composición de poblaciones de organizaciones se explica a partir de dos tipos fundamentales de determinantes: (1) los asociados a la propia dinámica endógena de las poblaciones bajo análisis y (2) los referidos a variaciones en el entorno donde las poblaciones están insertas (Carroll y Hannan, 2000). En la República Argentina se produjeron cambios contextuales de considerable magnitud: apertura indiscriminada de la economía, masivo retiro del Estado de la producción directa de bienes y servicios y establecimiento de la convertibilidad de la moneda local con el dólar, que se tradujo en la sobrevaluación dramática de la primera. Los efectos de estos cambios contextuales fueron devastadores en muchos sectores industriales del país. Siguiendo la analogía biológica, la emergencia de una masiva concentración económica en manos de firmas de capital extranjero y el tipo de cambio sobrevaluado produjeron un cambio ecológico catastrófico, similar al de la extinción de los dinosaurios. En este caso, los dinosaurios fueron las empresas de capital nacional, especialmente aquellas de menor tamaño, que son las que emplean la mayor proporción de la fuerza laboral (Aldrich, 1999).

La apertura de la economía y el nuevo clima empresarial de los noventa, fruto de la revolución menemista, contribuyeron a acercar inversión extranjera directa al país. En un mundo donde el capital comenzó a moverse más libremente, Argentina aparecía como un destino interesante para expandir líneas de negocios, especialmente cuando la privatización de empresas públicas presentaba interesantes oportunidades para quienes buscaban rentabilidad. Así, la penetración de empresas multi- 
nacionales en Argentina fue un rasgo central de la economía de los noventa. En 1994, 131 de las 200 mayores empresas del país, con ventas que representaban el $57 \%$ del total, estaban en manos de capitales argentinos. En 1998, esto se había invertido: 131 de esas 200 firmas, que concentraban el $70 \%$ de la facturación, estaban dominadas por capitales extranjeros (Guadagni, 2000). Para la República Argentina, la globalización fue esto: el pasaje de las mayores y más rentables compañías a manos de capital extranjero. Si bien algunos autores restan importancia a la extensión y novedad del fenómeno de la globalización (Sutcliffe y Glyn, 1999), uno de sus indicadores fundamentales es el nivel de penetración de empresas multinacionales en un país. Precisamente este indicador posibilita entonces afirmar que la economía argentina, con los enormes cambios en la estructura de propiedad empresaria, se convirtió en uno de los países más globalizados o abiertos al mundo en este período, en un proceso posibilitado por el ajuste estructural planeado por la élite política gobernante, apoyado por la burguesía local y favorecido por operadores económicos extranjeros y organizaciones multilaterales de crédito.

De acuerdo a Conesa (2000), la eliminación de las restricciones a la entrada de capitales extranjeros en un contexto de alta tasa de interés real, como fue la que caracterizó a la Argentina en la mayor parte de los años noventa, no sólo propició el ingreso de capitales especulativos, sino que implicó una limitación de acceso al crédito a empresas locales, especialmente pequeñas y medianas. En cambio, las empresas multinacionales establecidas en el país gozaron de una ventaja competitiva adicional, ya que podían obtener fondos a tasas más bajas en sus países de origen. La compra de empresas locales era una oportunidad de negocios que aparecía como tentadora para muchas firmas multinacionales, ya que podían adquirir posiciones oligopólicas en el mercado argentino, eliminando a sus competidores locales.

La extranjerización de la economía también respondió, además, a la adquisición de las empresas que fueron privatizadas. En tales casos, dado el enorme capital necesario involucrado en las transacciones, sólo empresas extranjeras estaban en condiciones financieras de afrontar la compra. A menudo, estas operaciones se realizaban en forma conjunta entre una firma extranjera y una local con el apoyo de grupos financieros internacionales. Por otra parte, las condiciones de negocio obtenidas eran verdaderamente favorables, en la medida en que el riesgo empresarial, tan alabado por el gobierno privatizador, había sido eliminado o reducido drásticamente en los contratos de adquisición. En tal sentido, en algunas operaciones se incluían cláusulas de reembolso por parte del Estado en el caso de pérdidas operativas e incluso se garantizaba la posibilidad de incrementos de precio, independientemente de los cambios producidos en la estructura de costos de las empresas privatizadas.

El nuevo perfil del modelo de acumulación de la economía argentina estaba caracterizado por una estructura de precios relativos que claramente favorecía al sector de productos no transables (los bienes inmuebles) en relación al de productos transables (los bienes muebles) (Chisari et al., 1996), debido a la sobrevaluación de la moneda local. Además, la reciente apertura de la economía llevó al país a un retorno a la especialización productiva en áreas vinculadas a los recursos naturales 
(Kosacoff, 2000a). Así, la tendencia en la composición de las exportaciones marcaba una declinación en el valor agregado de los productos. La producción industrial se vio naturalmente afectada por este giro hacia la reprimarización de la economía, que fue caracterizado como una inserción pasiva en el comercio internacional (Sevares, 1997). Según Kosacoff (2000b: 191), "existe un amplio consenso en los investigadores de la industria respecto a que en los años noventa, la actividad manufacturera fue, en términos netos, perdedora de empleo", destacando además que el número de establecimientos industriales disminuyó en la citada década. Así, la importancia de la industria como motor del crecimiento muestra un declive progresivo. En contraposición a ello, el sector servicios incrementó su participación en el PBI, dado el desplazamiento de inversiones hacia el sector de bienes no transables de la economía.

Al igual que en la economía real, en los noventa el sector financiero fue gradualmente cayendo en manos del capital extranjero. De hecho, la política de apertura económica hacia la inversión externa, basada en una creencia de un carácter casi religioso respecto a la misión salvífica del capital extranjero no sólo inducía la entrada de inversiones productivas, sino especulativas. En este último caso, las consecuencias suelen ser negativas, dado el divorcio entre la economía real y la financiera (Scavo, 1996). El sistema bancario experimentó un proceso de concentración y extranjerización a partir de la crisis del efecto tequila en 1994, que culminó en el año 2000 con más del 50\% de los depósitos del sistema bancario en manos de entidades financieras del exterior. ${ }^{3}$

Aunque una parte importante de la renta de las empresas multinacionales retorna a sus países de origen, puede pensarse que parte de esta renta es reinvertida en el mercado local, generando empleo y contribuyendo a incrementar la riqueza del país en que se radican las inversiones. No obstante, esto no ocurrió en el caso argentino. En teoría, la inversión extranjera directa puede producir un efecto positivo sobre una economía doméstica si se dirige a sectores que generan valor agregado, pero el modelo de desarrollo capitalista que seguía la Argentina impedía esto. La inversión extranjera se orientaba a obtener ganancias en el sector no transable de la economía (en servicios como telecomunicaciones, finanzas, turismo, etc.) y en la producción de bienes destinados principalmente al mercado interno. Por otra parte, siguiendo los patrones sugeridos por los modelos de downsizing de moda en los países centrales de sus casas matrices, una de las primeras medidas adoptadas por las firmas extranjeras fue la reducción de personal en las compañías locales adquiridas. Además, la concentración industrial que paulatinamente abarcaba a los diversos sectores de actividad generaba quiebras en los competidores más pequeños, que son quienes hacen un uso más extensivo de mano de obra con relación al capital. En definitiva, la extranjerización de la economía fruto de la combinación del ajuste estructural y la apertura económica, un contexto de liquidez internacional y una tasa de cambio

\footnotetext{
${ }^{3}$ Véase Clarín (2000a).
} 
sobrevaluada convirtió a los argentinos "en meros inquilinos en su propio país" (Conesa, 2000:33).

\section{Las consecuencias sociales del ajuste: desempleo, desigualdad y criminalidad}

El plan de estabilización tuvo éxito en materia de reducción de la inflación (Tabla 2). Sin embargo, aunque a partir de la segunda mitad de los noventa la inflación alcanzó mínimos históricos, ocurrió otra cosa en los primeros años del plan, cuando el componente inercial de la inflación continuó operando y contribuyó a un alza de precios que produjo una sobrevaluación de la moneda local contra el dólar. La inflación es considerada un impuesto altamente regresivo porque afecta a todos los individuos independientemente de la riqueza que posean. Por lo tanto, su reducción resultó beneficiosa para los más pobres. La sobrevaluación del tipo de cambio, además, hizo posible un incremento de importaciones tanto de bienes de consumo como de capital. Además, los salarios nominales en dólares aumentaron. Esto produjo un boom de consumo durante los primeros años del Plan de Convertibilidad, que contribuyó a legitimar la política económica a los ojos de diversos sectores de la población. Para la propaganda oficialista, esto no era sino una demostración del éxito del programa económico y de la entrada de la Argentina al Primer Mundo. Esto es consistente con la propaganda oficialista de Peter Berger (1986), quien sostiene que la base legitimante del capitalismo es su capacidad para proveer bienes y servicios a los ciudadanos. El consumismo, según este autor, es en última instancia la fuerza legitimante del sistema, una fuerza que además requiere de mecanismos ideológicos de control.

\section{Tabla 2 - Inflacion anual en la Republica Argentina (1990-2001)}

\begin{tabular}{|l|c|}
\hline Año & Tasa de inflacion \\
\hline 1990 & $1.343,9$ \\
\hline 1991 & 84,0 \\
\hline 1992 & 17,5 \\
\hline 1993 & 7,4 \\
\hline 1994 & 3,9 \\
\hline 1995 & 1,6 \\
\hline 1996 & 0,1 \\
\hline 1997 & 0,3 \\
\hline 1998 & 0,7 \\
\hline 1999 & $-1,8$ \\
\hline 2000 & $-0,7$ \\
\hline 2001 & $-1,5$ \\
\hline
\end{tabular}

Fuente: elaboración propia en base a datos de Cepal (2011b) 
Sin embargo, tres aspectos destacan como problemáticos en la Argentina de los años noventa: el desempleo, la criminalidad y la creciente desigualdad social. Los dos primeros surgían más claramente en las encuestas sobre la preocupación de los ciudadanos. ${ }^{4}$ La desigualdad no figuraba como una preocupación prominente, pero indudablemente fue uno de los rasgos distintivos del período. En rigor, estos problemas sociales no podían resultar inesperados. El modelo económico de la convertibilidad al impulsar la sostenida apreciación del tipo de cambio produjo un efecto que era predecible: la sustitución de mano de obra manual (cuyo costo en dólares había aumentado) por capital (Beker, 1998). Además, también por efecto del problema cambiario, muchas industrias locales se tornaron inviables, ya que resultaba más barato adquirir bienes importados. Ambos factores, sustitución de mano de obra por capital y cierre de numerosas pequeñas y medianas empresas, condujeron a un aumento en la tasa de desempleo, que alcanzó un valor de 18,45 en mayo de 1995. Como puede observarse en la Figura 2, los niveles de desempleo no fueron circunstanciales sino que representaron un alza en el desempleo estructural, duplicando o triplicando los valores históricos tradicionales, debido a las profundas modificaciones inducidas en la estructura productiva del país a partir del elevado tipo de cambio, que era la piedra fundamental del plan económico.

${ }^{4}$ Por ejemplo, “Orden y seguridad, dos reclamos que crecen” (Clarín, 2000b). 
Figura 2 - Tasa de Desempleo (1982-2007)

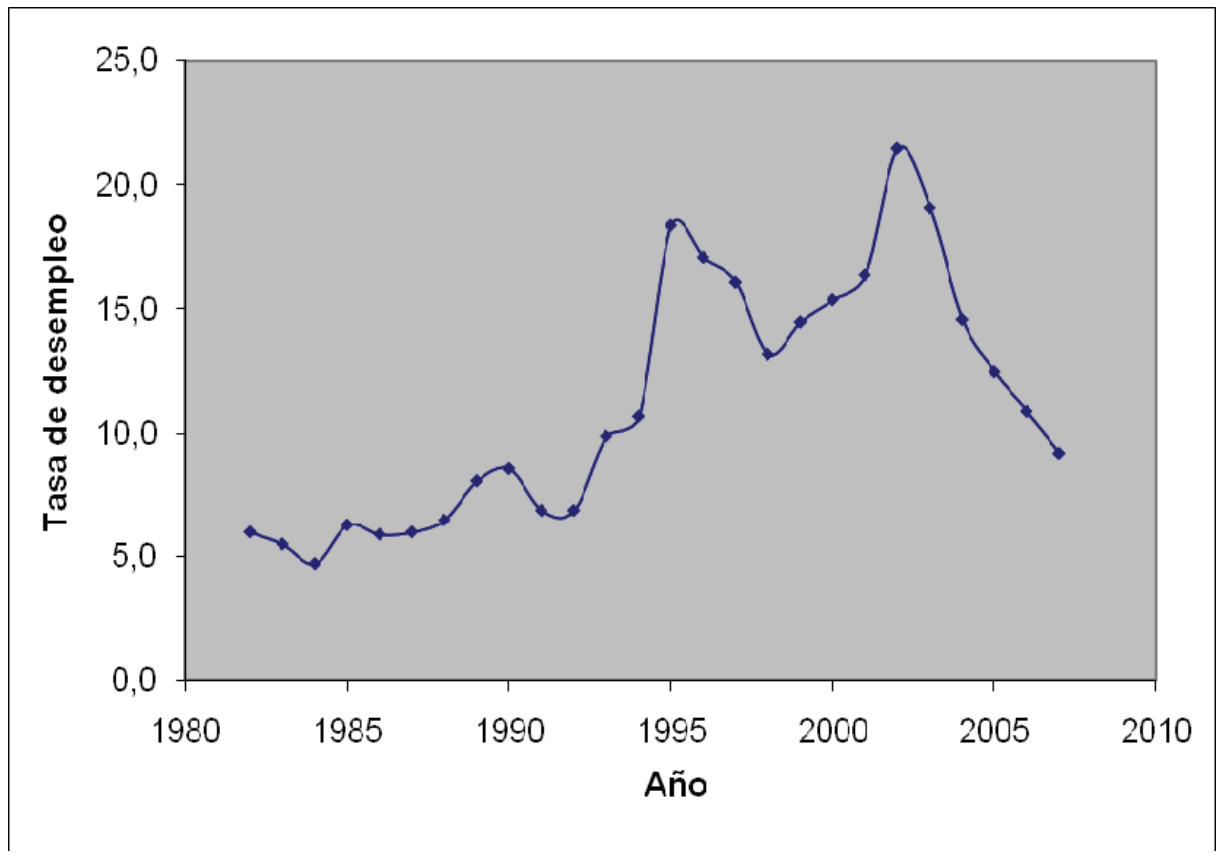

Nota: los valores de los años 1982 a 2002 corresponden a datos a mayo del año de referencia, los valores de 2003 en adelante corresponden a datos del primer semestre

Fuente: elaboración propia en base a datos de la Encuesta Permanente de Hogares del INDEC (2011a, 2011b)

Las privatizaciones coadyuvaron de algún modo al aumento del desempleo, ya que los compradores de las empresas privatizadas realizaron reducciones en las plantas del personal. Como se señaló en la sección anterior, también las firmas locales adquiridas por empresas multinacionales sufrieron reducción de personal. La mayor parte de los desempleados, no obstante, provinieron de la masiva ola de quiebras de empresas pequeñas y medianas, que no pudieron adaptarse a los cambios estructurales. ${ }^{5}$ Claramente, las firmas surgidas al amparo del modelo de sustitución de importaciones no lograron llevar a cabo los cambios necesarios para competir en una economía abierta con un tipo de cambio tan sobrevaluado. Aunque

5 Esto incluso se reflejaba en los debates periodísticos de la época. Véase, por ejemplo, Kohanoff (2000). 
el desempleo disminuyó en 1996, debido a un programa gubernamental de estímulo a la creación de empleos precarios, no alcanzó los niveles de la década previa.

De la mano del desempleo, se hizo evidente una tendencia de creciente desigualdad en la distribución del ingreso. Ambos factores pueden estar causalmente asociados (Hoover et al., 2009), ya que una disminución importante del empleo puede afectar más severamente a los sectores de menores ingresos de la población. Como puede observarse en la Tabla 3, los coeficientes Gini, que miden el nivel de desigualdad social, muestran una tendencia ascendente desde 1991. El discurso oficial se apoyaba, en mayor o menor medida, en el llamado "efecto derrame", noción que puede derivarse de la formulación de Kuznets (1955), que postulaba una relación de U invertida entre desarrollo e igualdad en la distribución de la renta. Los aumentos iniciales del nivel de desarrollo inducían un crecimiento en la desigualdad, hasta que se alcanzaba un máximo a partir del cual incrementos posteriores del desarrollo contribuían a generar una distribución más equitativa. Hallazgos más recientes cuestionan esta relación, sugiriendo que el vínculo entre desarrollo económico y equidad social es, en realidad, más complejo (Bourguignon, 1998). La evidencia empírica de los países latinoamericanos falsifica la teoría del efecto derrame del crecimiento económico. Sin embargo, en la medida que una estrategia de control ideológico, que en ocasiones recurría al discurso de lo inevitable (Therborn, 1988), posibilitaba la tolerancia a este aumento en la desigualdad, la clase dominante no tenía motivos para alarmarse. Por otra parte, debe señalarse que las políticas de ajuste estructural enfrentaron una escasa o nula resistencia por parte de la clase trabajadora. Esto último era esperable ya que el partido gobernante, el justicialismo, tenía firmes vínculos con el movimiento sindical, lo que permitió una estrategia de cooptación de sus principales líderes (Murillo, 2005) ${ }^{6}$

\footnotetext{
${ }^{6}$ La afinidad política y los lazos personales entre el presidente Menem y los principales líderes sindicales anularon o limitaron los escasos intentos de resistencia organizada de los trabajadores contra la implementación de las políticas de ajuste (Levistky y Way, 1998). En cambio, con el gobierno radical elegido en 1999, se verificó cierto nivel de resistencia obrera, con la extraña connivencia de miembros del partido Justicialista.
} 


\section{Tabla 3 - Evolucion de la desigualdad en la Republica Argentina - estimaciones del indice de Gini (1986-2009)}

\begin{tabular}{|c|c|}
\hline Año & Indice de Gini \\
\hline 1986 & 44,51 \\
\hline 1992 & 45,35 \\
\hline 1996 & 48,58 \\
\hline 1998 & 49,84 \\
\hline 2002 & 52,52 \\
\hline 2004 & 51,28 \\
\hline 2005 & 50,03 \\
\hline 2006 & 48,81 \\
\hline 2009 & 45,77 \\
\hline
\end{tabular}

Fuente: elaboración propia en base a datos del Banco Mundial (2011)

Otro de los desarrollos más preocupantes del período fue el notorio incremento de los índices de criminalidad, especialmente en los crímenes contra la propiedad (Figura 3) Entre los potenciales factores explicativos, algunos autores hacen mención al aumento del desempleo (Cerro y Meloni, 1999). El incremento en la desigualdad de ingresos ha sido también mencionado como un potencial determinante de la criminalidad (Patterson, 1991). Por otra parte, la adopción de nuevos valores socioeconómicos propios de la etapa de globalización del capitalismo (gratificación instantánea, consumismo) también puede en parte dar cuenta de la creciente ola de criminalidad (Taylor, 1999). Además, como factores que favorecen el auge del crimen, se deben incluir la mayor facilidad para obtener armas y el incremento en el consumo de drogas, todos ellos presentes en la Argentina de ese momento (y en la actualidad, naturalmente) y que se asocian a la expansión del capitalismo, o más precisamente de las facetas ilegales de la sociedad de fin de milenio (Castells, 1998). Si la República Argentina en algo comenzó a parecerse más a los países desarrollados durante los noventa fue especialmente en el aumento de la criminalidad, especialmente entre los individuos de menor edad. El tema ha sido mencionado como una de las mayores preocupaciones de la población, junto con el desempleo, siendo debatido como uno de los temas centrales de las campañas políticas. 
Figura 3 - Delitos con intervencion policiaca (1991-2008)

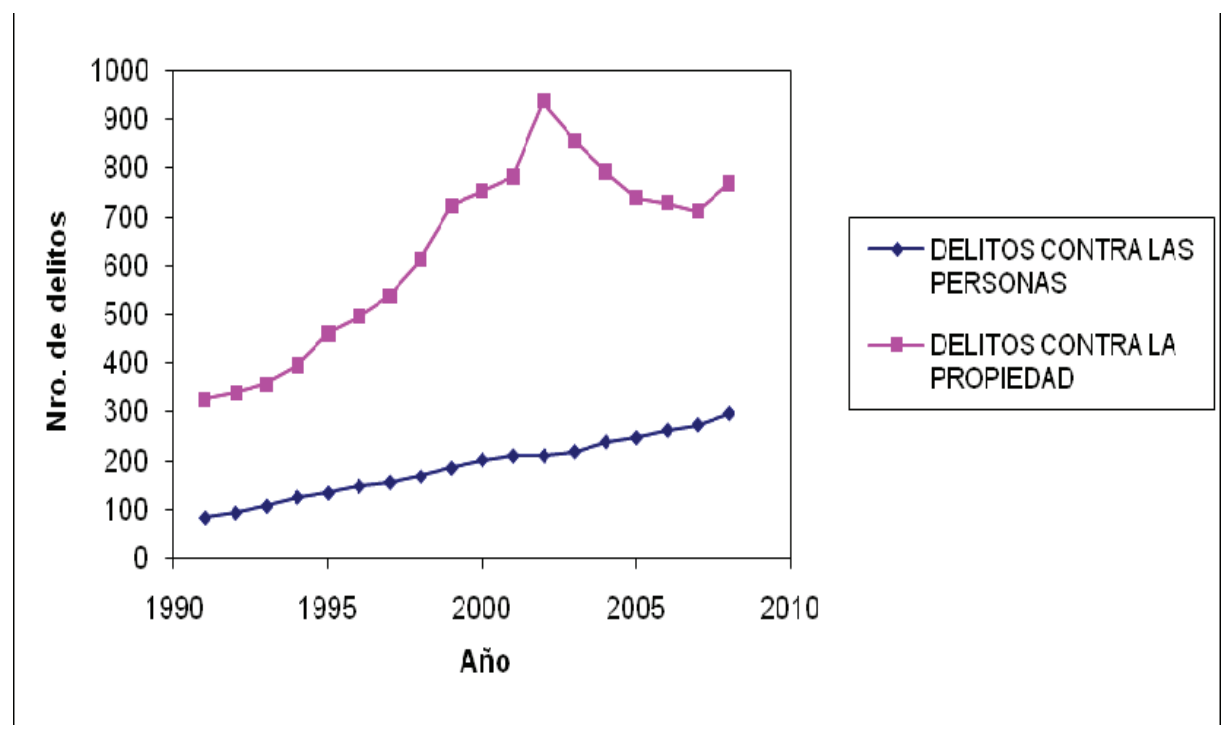

Nota: número de delitos en miles

Fuente: elaboración propia en base a datos de la Dirección Nacional de Política Criminal (2011)

El inventario de consecuencias adversas incluye otros elementos como el deterioro de la inversión en educación y salud. La relación entre estos aspectos y el ajuste estructural es clara. A medida que aumenta el peso de los servicios de la deuda, sus intereses y amortizaciones, hay menos fondos disponibles para otros fines. El imperativo de la reducción del gasto público, demandado por los organismos multilaterales de crédito, hizo que el gobierno recurriese al aumento de los impuestos al consumo, de fácil recaudación pero netamente regresivos, con los cuales la mayor presión siempre recae sobre los que menos tienen. No obstante, en una suerte de círculo vicioso, el incremento impositivo se volvió insuficiente, especialmente con la desaceleración del crecimiento en 1999. Entonces, fueron necesarios recortes en los salarios a los empleados públicos, lo que produjo un incremento de la conflictividad laboral dentro del sector público. Basta indicar al respecto que a inicios del 2000 , el monto pagado en concepto de intereses y amortizaciones de la deuda externa había finalmente sobrepasado a los gastos abonados por salarios de empleados públicos, ya que el primer concepto se había incrementado, mientras el segundo permanecía casi constante. A modo de comparación, en 
1993 por cada peso que el Estado abonaba a los acreedores externos, se pagaban 2,3 pesos en concepto de salarios. ${ }^{7}$

Esta breve descripción muestra claramente cómo el impacto de la globalización y la apertura económica, que en la práctica significaron estrictamente la extranjerización de la economía, estuvieron acompañados de aspectos negativos que afectaron especialmente a los sectores más desprotegidos de la sociedad.

\section{El colapso y su salida}

En el año 2001, la recesión golpeó duramente a la economía argentina. Eran los primeros signos de que la Convertibilidad estaba llegando a sus momentos finales. Los operadores económicos percibieron claramente la fragilidad de las finanzas públicas con un déficit preocupante, especialmente debido a la dificultad para hacer frente a los servicios de amortización e intereses de la deuda pública. Comenzó así una fuga de capitales. Era necesario reinstalar la confianza en el gobierno, pero esto no podía llevarse a cabo si no se daban las señales adecuadas de que se produciría un ajuste fiscal. En marzo de 2001, para calmar a los mercados se eligió como Ministro de Economía a Ricardo López Murphy, un economista con estudios en la Universidad de Chicago, percibido como confiable por el establishment. Al asumir, López Murphy planteó claramente que serían necesarios sacrificios para mantener el rumbo económico y propuso una reducción de salarios de los funcionarios públicos. La medida fue severamente criticada por sectores de la oposición e incluso de la propia alianza de partidos en el poder, y López Murphy renunció inmediatamente luego de su designación.

Una figura de relevancia ocupó su lugar, el arquitecto del Plan de Convertibilidad, Domingo Cavallo. Con nivel informal de "superministro" durante la gestión de Menem y luego de haber fundado un partido político con el cual disputó las elecciones presidenciales en 1989, regresaba a la función pública también con un aura de superministro. Los mercados esta vez reaccionaron con cautela. Cavallo tenía el difícil objetivo de acallar la tempestad en ciernes. Una de sus iniciativas fue una renegociación de la deuda externa para alejar el fantasma del default inminente. No obstante, la fuga de capitales continuaba. Con el objeto de evitar una crisis sistémica del sector financiero, Cavallo dispuso medidas que impedían a la gente retirar sumas en efectivo de los bancos, cuando éstas superaran un cierto nivel mínimo. Fue el comienzo del fin. En la práctica, este intento de bancarización forzosa, popularmente denominado "corralito", irritó por igual a las clases medias y a los sectores más desprotegidos de la población. Comenzaron manifestaciones ciudadanas de diversa índole reclamando la renuncia del presidente De la Rúa. Esta presión popular, a la que debe añadirse como factor más relevante la falta de respaldo

\footnotetext{
${ }^{7}$ Véase Clarín (2000c).
} 
político por parte no sólo de la oposición justicialista sino de sectores del propio partido radical, culminó en la renuncia del mismo.

Luego del rápido tránsito de varios presidentes provisionales, se hizo cargo de la presidencia Eduardo Duhalde, uno de los líderes del justicialismo. Su tarea de gobierno no fue sencilla ya que afrontaba una crisis sin precedentes históricos. En primer lugar, debió llevar a cabo la inevitable devaluación de la moneda, cuyos efectos sobre los salarios de los trabajadores se hicieron sentir. La pobreza subió a niveles record, así como el desempleo, que como se aprecia en la Figura 2 alcanzó su pico histórico en el año 2002, ya que la incertidumbre respecto al futuro de la economía era tal que paralizaba cualquier posibilidad de inversión. Además, las limitaciones para operar dentro del sistema financiero seguían vigentes, por miedo a una quiebra general del mismo. Con el transcurso de los meses, la economía comenzó a funcionar de nuevo. El peso devaluado inyectó competitividad a la economía. Además, el incremento de los precios internacionales de algunos commodities agrarios favoreció a los productores de los mismos. Crecieron así las exportaciones y el gobierno se benefició por contar con recursos financieros adicionales, a partir del establecimiento de retenciones a las exportaciones. Estaban dadas las condiciones para que la economía argentina arrancara de nuevo, previsiblemente con un efecto rebote, como suele ocurrir después de una crisis.

Atravesado el momento más difícil y recuperada la calma institucional, Duhalde convocó a elecciones presidenciales. Tras nuevas elecciones presidenciales, en mayo de 2003 asumió como presidente otro político justicialista, Néstor Kirchner, quien continuó inicialmente los lineamientos de política económica de Duhalde, conservando incluso al Ministro de Economía designado por este último. Más adelante, Kirchner designó a otro funcionario para cubrir dicha cartera y gobernó con mayor personalismo en una línea de política que puede considerarse de centroizquierda. Gozando de una coyuntura internacional extremadamente favorable por el sostenido incremento del precio de los commodities, su gobierno produjo cierta mejora en los indicadores económicos. El crecimiento fue sostenido, excepto en el año 2009 por causa de la crisis financiera internacional (Tabla 1), y el desempleo descendió paulatinamente (Figura 2). El default de la deuda externa y la posterior renegociación de parte de ésta hicieron que los indicadores de endeudamiento mostrasen un perfil más favorable que durante el gobierno menemista.

En 2007, Kirchner fue sucedido como presidente por su esposa, quien siguió adelante con la misma política. Si bien en política exterior ha existido una cercanía del gobierno argentino con Hugo Chávez, el dirigente venezolano de corte populista que impulsa un modelo de socialismo del siglo XXI, los Kirchner no han avanzado en un proyecto estatista tan radical como el propugnado por Chávez. No obstante, hubo un retroceso en la extranjerización de la economía. Algunos bancos extranjeros abandonaron el país y la inversión extranjera directa ya no percibe a Argentina como un destino atractivo, al menos en relación con otros países de la región como Brasil y Chile. Incluso, la retórica dirigista de los Kirchner y algunas de sus iniciativas económicas han motivado que empresarios argentinos se radicasen en otros 
países de la región, en los cuales creen encontrar condiciones más favorables para la libre empresa.

En lo social, se observan algunos avances. Como se aprecia en la Tabla 3, la desigualdad en el ingreso, que alcanzó su punto máximo en el año 2002, en el cual la devaluación afectó con más fuerza los ingresos reales de la mayoría de la población, comenzó a retroceder alcanzando niveles similares a los de inicios de la década del noventa. En este sentido, es pertinente señalar que el gobierno de los Kirchner realizó una política de asistencialismo que benefició a los sectores de menores ingresos. Sin embargo, de cara al futuro, subsisten dudas respecto a la viabilidad del modelo económico adoptado, que en última instancia se basa en unos pocos sectores con ventajas competitivas (el sector agrícola) o que se nutren de la demanda del principal socio del Mercosur, Brasil (en particular, la industria automotriz).

El problema es nuevamente de índole fiscal. El endeudamiento externo es menor que en la época de Menem pero con tendencia a incrementarse. La inflación surge nuevamente como una amenaza en el horizonte. La balanza comercial, muy favorable inicialmente luego de la devaluación y por el incremento de los precios de los commodities, exhibe un saldo positivo pero en descenso. Por otra parte, las ventajas de la devaluación se agotan paulatinamente y la industria local pierde aceleradamente competitividad. Con precios en alza y esquemas asistencialistas que requieren una gran cantidad de fondos, el Estado podría quedarse desfinanciado si su capacidad recaudatoria disminuye, por ejemplo, ante la pérdida impositiva causada por un descenso en los commodities. Indudablemente, el populismo de centro izquierda implementado por el gobierno de los Kirchner, con su intento de revitalizar el modelo de sustitución de importaciones, podría agotarse al igual que lo hizo en épocas de otros gobiernos. Los incrementos de la inflación en 2009 y 2010 son un primer indicador de que el modelo elegido atraviesa problemas. ${ }^{8}$ No obstante, es altamente improbable que se llegue a repetir una crisis de magnitud similar a la del año 2001.

\section{Discusión y conclusiones}

Después de una década de ajuste estructural, la República Argentina dejó atrás el estatismo, recuperó algunas instituciones capitalistas y logró una nueva inserción en el capitalismo global. Más allá de la euforia inicial de los tecnócratas amigos del poder y los burócratas de organismos internacionales, los resultados fueron, a la postre, negativos. El país terminó con una deuda externa muy superior a la del

${ }^{8}$ Existen dudas sobre los datos oficiales sobre el índice de inflación proporcionados por el INDEC (Instituto Nacional de Estadística y Censos), a partir de su intervención en 2007. Véase, por ejemplo, Lindenboim (2007). 
comienzo de la década, pese a los ingresos extraordinarios provenientes de su ambicioso plan de privatizaciones. La estructura económica involucionó del agotado modelo de sustitución de importaciones a una suerte de reprimarización. El desempleo se incrementó al igual que la desigualdad y el índice de criminalidad. Si bien la inflación llegó a ser controlada, el mercado interno se redujo, excepto en ciertas áreas específicas de bienes no transables, cuyo target es la clase de mayor poder adquisitivo. Las mayores empresas de la economía pasaron a manos extranjeras y las PYME que subsistían afrontaron notables dificultades de financiamiento, ya que el crédito bancario estaba orientado básicamente al Estado, las grandes empresas y las personas físicas (Angelelli y Moori-Koenig, 1999). En suma, las pérdidas superaron los escasos beneficios de las políticas adoptadas, muy reducidos estos últimos y concentrados en los sectores altos de la sociedad aliados a los poderosos intereses extranjeros cuyo peso en la economía resultaba mucho mayor que en el pasado.

¿Qué funcionó mal? Debe admitirse que las políticas llevadas a cabo resultan incluso contradictorias con el mismo diagnóstico del consenso de Washington, dado que dos elementos básicos del mismo fueron masivamente desoídos: (1) evitar la sobrevaluación del tipo de cambio y (2) reducir el déficit público. ¿Cómo pudieron cometerse errores tan garrafales, incluso con el visto bueno de los organismos financieros multinacionales y la banca extranjera? Algunos analistas advirtieron desde el inicio que el anclaje del tipo de cambio en una paridad sobrevaluada sería finalmente desastroso (Conesa, 2000; Muchnik, 1993). Sin embargo, los tecnócratas de turno descartaron la idea misma de que la moneda pudiese estar sobrevaluada (Pastor y Wise, 1999). Antes bien, ante la evidencia empírica incontrastable de un cambio en la estructura de precios relativos en dirección a un incremento de precio en divisas de los bienes no transables, explicaron el fenómeno apelando a las leyes de oferta y demanda. Argumentaron que al abrirse Argentina a un mundo globalizado, despreciando el semiestatismo de décadas anteriores y abrazando la disciplina del libre mercado, los capitales extranjeros comenzarían a percibir que el país encerraba una oportunidad de negocios en sectores tales como los servicios, la construcción, la hotelería, las finanzas, y muchos otros. Este redescubrimiento de Argentina por el "norte civilizado y civilizador" era el factor detonante del incremento de precios y, consistentemente, de los salarios en dólares. La reprimarización de la economía no era un dato que preocupara, sino otra consecuencia de la operación de los mecanismos de oferta y demanda a nivel internacional. Dicha situación se revertiría en cuanto el país alcanzase mayor desarrollo en términos de capital humano. Tal vez con doble discurso, de acuerdo a Scavo (1996), los técnicos del Banco Mundial y el FMI avalaron semejante diagnóstico. Los policy makers del poder y el mismo presidente (Menem, 1999) sostenían que Argentina había entrado en la sociedad del conocimiento donde los premios y recompensas eran función del grado de conocimientos de la fuerza laboral del país. Se reconocía que el desarrollo era impulsado por la exportación de bienes con alto valor agregado y ello sólo era posible en el contexto de una nación que privilegiase el capital humano de su fuerza laboral. 
Explicaciones que dan cuenta de estos resultados exclusivamente sobre la base de la sobrevaluación del tipo de cambio y el incremento del déficit público, aunque acertadas, están planteadas a un nivel epifenoménico, ya que son precisamente estos aspectos los que necesitan ser explicados. La clave del análisis reside en interpretar las políticas llevadas a cabo, más que como un error grosero de política económica, como una suerte de metapolítica de inserción en la economía mundial, que resultó funcional a la presente etapa de expansión del capitalismo, tanto en la economía real como en la megaespeculación financiera. Así, podemos entender que el anclaje del dólar operó como un seguro de cambio que atrajo tanto a capitales financieros y otros destinados a inversiones reales. Esta hipótesis postula un vínculo de completa subordinación de la política económica en países periféricos a la lógica de favorecer intereses externos. La extranjerización no fue sino el rostro de la globalización capitalista en países periféricos. El caso argentino, con el extraño lecho de Procusto del Plan de Convertibilidad, fue quizás un ejemplo caricaturesco, pero no por ello menos ilustrativo de las consecuencias nefastas que puede acarrear una inserción subordinada a la economía global. En términos comparativos, al analizar otros casos nacionales deberían considerarse dimensiones analíticas como el nivel de autonomía de la clase política local, la importancia de la economía nacional en cuestión, la existencia de núcleos de resistencia, el compromiso de la burguesía local con la economía de su país, etc.

En el caso bajo análisis, un elemento explicativo clave en la elección del modelo de desarrollo e inserción en la economía mundial (el debt-led growth) fue la actitud de la clase política local, altamente compatible con las pretensiones e intereses de la burguesía local, que abrazó el proyecto extranjerizante de la economía, prefiriendo obtener superganancias a través de la venta de empresas a capitales extranjeros, permutando capital físico por capital financiero, y la asociación con grupos multinacionales para explotar servicios privatizados (Katz, 1993; Schvarzer 1998). La convertibilidad, por otra parte, les permitió una extraordinaria valorización de sus activos y un flujo de ingresos en divisas como retorno sobre activos, que difícilmente podrían haber obtenido en otras condiciones. Los economistas y consultócratas, en su casi absoluta mayoría también defendieron el plan elaborado, obteniendo magníficos ingresos en dólares que hubieran sido imposibles de lograr en otro contexto. Incluso los pocos economistas no contestatarios que criticaron el modelo recurren a explicaciones sociológicas cualitativas de esta índole para explicar su incomprensible pervivencia y soporte por parte de los organismos multilaterales de crédito. Conesa (2000: 3), por ejemplo, afirmó que la política económica era apoyada por ciertos "intereses creados", entre los cuales estaban "los economistas locales ligados al debt-led growth model y a la supply side economics, es decir, al negocio de las comisiones de la deuda externa y de las "privatizaciones", que tan buena prensa tuvieron en el período 1991-99"

El caso argentino ha sido presentado como un ejemplo paradigmático de los problemas del neoliberalismo (Harvey, 2005; Steger y Roy, 2010), pero en rigor lo que puso de manifiesto no es la brecha entre las promesas del discurso neoliberal y sus logros, sino cómo los defensores del mercado libre y el Estado mínimo pueden en la 
práctica aplicar en forma muy selectiva los propios cánones de su doctrina. Es cierto que los planes de ajuste estructural en los años noventa fueron adoptados por muchos países en una suerte de convergencia de políticas (Henisz et al., 2005), pero las clases dominantes locales tuvieron un cierto margen de libertad en la implementación de este gran diseño, como lo demuestra Murillo (2009) en el caso de América Latina. En mi opinión, en el caso argentino, el discurso neoliberal convivió con las tendencias populistas del partido gobernante y esta combinación, con resultados inicialmente positivos, no resultó finalmente sostenible.

En diversos países de la región, los años noventa asistieron a la aparición de un fenómeno nuevo, la aparición de líderes con gran apoyo popular que orientaron sus políticas públicas en torno al libre mercado y a un replanteamiento del rol del Estado. Esto se denominó neopopulismo para diferenciarlo de una primera fase del populismo marcada por un discurso nacionalista y estatista (Weyland, 2003). Analíticamente, la similitud entre ambos tipos de populismo radica en la movilización de apoyo de sectores populares, típicamente marginados de la política, como forma de acceso al poder. Sin embargo, en Argentina, el elemento populista estuvo marcado por la inflexibilidad en reducir el gasto del Estado, lo que era necesario en virtud de la propia lógica del programa económico. Como señala Mussa (2002: 15), el motivo central de la tragedia fue "la inhabilidad crónica de las autoridades argentinas de mantener una política fiscal responsable". Las transformaciones estructurales del Estado, aunque de magnitud, fueron simplemente un cambio en su perfil de funciones, pero no constituyeron una reducción significativa del déficit del sector público. El personal del Estado nacional decreció notablemente, pero por transferencia de agentes a niveles subnacionales. De hecho, lejos de disminuir, el gasto público consolidado en dólares constantes aumentó 57,5\% entre 1999 y 1990 (Oszlak, 2003). De esta forma, el colapso era inevitable. El modelo de capitalismo adoptado en Argentina, que inevitablemente generaba mayores demandas sobre el Estado, terminó produciendo una crisis fiscal (O'Connor, 1973). Irónicamente, fue un gobierno de centro-izquierda el que tuvo que poner las cuentas en orden con una política fiscal más sustentable en los años inmediatos a la crisis. Hasta cuándo podrá mantenerse la misma es un interrogante abierto. Al presente, el contexto internacional por vía del comercio exterior permitió al Estado argentino obtener una fuente genuina de financiamiento, pero no es posible precisar cuánto puede prolongarse dicha situación o, dicho de otro modo, cuándo puede sobrevenir la próxima crisis.

\section{Referencias bibliográficas}

Aldrich, H. E. (1999). Organizations evolving. London: Sage.

Angelelli, P. y Moori-Koenig, V. (1999). “Acceso al financiamiento”. En G. Yoguel y V. Moori-Koenig (eds.), Los problemas del entorno de negocios. El desarrollo competitivo de las PyMEs argentinas. Miño y Dávila Editores: Buenos Aires. 
Banco Mundial (2011) World Development Indicators, http://data.worldbank.org/data-catalog (con acceso 22.05.2011)

Beker, V. (1998). "Globalization and unemployment: The case of Argentina". Trabajo presentado al XX Congreso de la Latin American Studies Association, Chicago, USA.

Berger, P. (1986). The capitalist revolution: Fifty propositions about prosperity, equality, and liberty. New York: Basic Books.

Bourguignon, F. (1998). "Inequality and economic growth". En S.J.Burki, S.Aiye y R.Hommes (eds), Annual World Bank conference on development in Latin America and the Caribbean 1996: Poverty and inequality. Washington: World Bank.

Carroll, G. y Hannan, M. (2000). The Corporate demography of organizations and industries, Princeton: Princeton University Press.

Castells, M. (1998). End of millennium, The information age: Economy, society and culture, Vol. 3. Oxford: Basil Blackwell.

Cerro, A. y Meloni, O. (1999). "Distribución del ingreso, desempleo y delincuencia en Argentina". Trabajo presentado al XXXIV Congreso de la Asociación Argentina de Economía Política, Rosario.

Chisari, O., Fanelli, J. y Frenkel, R. (1996). “Argentina: growth resumption, sustainability and environment". World Development 24(2): 227-240.

CEPAL Oficina en Buenos Aires (2011a). Base de Indicadores Macroeconómicos. Precios Relativos, http://www.eclac.org/argentina/noticias/paginas/9/9839/Cuadro13.xls (con acceso 22.05.2011)

CEPAL Oficina en Buenos Aires (2011b). Base de Indicadores Macroeconómicos.

Precios Relativos.

http://www.eclac.org/argentina/noticias/paginas/9/9839/Cuadro12.xls (con acceso 22.05.2011)

Clarín (2000a). "El 51\% de los depósitos está en manos de bancos extranjeros". 20.02.2000: 28 .

Clarin (2000b). "Orden y seguridad, dos reclamos que crecen”. 07.25.2000: 6.

Clarín (2000c). "El Estado paga más intereses de la deuda que salarios públicos". 20.06.2000: 20 
Conesa, E. (2000). Qué pasa en la economía argentina, Buenos Aires: Macchi.

Dirección Nacional de Política Criminal (2011). Sistema Nacional de Informacion Criminal. Año 2008. Informe Argentina. Buenos Aires: Ministerio de Justicia, Seguridad y Derechos Humanos de la República Argentina.

Echegaray, F. (1996). "Condiciones económicas y preferencias electorales en Argentina, Perú y Uruguay”. Sociedad 10:57-102.

Edelstein, J. y Chilcote, R. (1986). Latin America: Capitalist and Socialist perspectives of development and underdevelopment, Boulder: Westview.

Feitlowick, M. (1999). A Lexicon of Terror: Argentina and the Legacies of Torture. Oxford: Oxford University Press.

Gerchunoff, P. y Cánovas, G. (1995). "Privatizaciones en un contexto de emergencia económica". Desarrollo Económico 35(136): 483-512.

Gerchunoff, P. y Torre, J.C. (1996). "La política de liberalización económica en la administración de Menem”, Desarrollo Económico 36(141): 733-768.

Graziano, W. (1990). Historia de dos hiperinflaciones: de Sourrouille a Erman González, Buenos Aires: Fundación Gabriel y Darío Ramos.

Griffith-Jones, S. y Sunkel, O. (1987). La crisis de la deuda y del desarrollo en América Latina: el fin de una ilusión. Buenos Aires: Grupo Editor Latinoamericano.

Guadagni, A. (2000). Contradicciones de la modernización. Buenos Aires: Centro de Estudios Estratégicos.

Harvey, D. (2005) A brief history of neoliberalism. Oxford: Oxford University Press.

Henisz, W., Zelner, B. y Guillén, M. (2005) "The Worldwide diffusion of marketoriented infrastructure reform 1977-1999". American Sociological Review 70(6): 871-897.

Hoover, G., Giderman, D. y Dibooglu, S. (2009) "Income inequality and the businesscycle: A threshold cointegration approach”. Economic Systems 33(3): 278-292

INDEC (2011a). Tasas de actividad, empleo, desocupación y subocupación correspondientes al total de aglomerados urbanos desde 1974 en adelante http://www.indec.gov.ar/nuevaweb/cuadros/4/shempleo1.xls (con acceso 22.05.2011) 
INDEC (2011b). Tasa de actividad, empleo, desocupación y subocupación por regiones y aglomerados desde el primer semestre de 2003 en adelante http://www.indec.gov.ar/nuevaweb/cuadros/4/sh_eph_continuasemestral.xls (con acceso 22.05.2011)

Katz, C. (1993). Economía latinoamericana. De la década pérdida a la nueva crisis, Buenos Aires: Letra Buena.

Kohanoff, R. (2000). "La industria ha sido destruída", Clarín, 02.18.2000, p. 17.

Kosacoff, B. (2000a). "The response of transnational corporations". En Bernardo Kosacoff (ed.), Corporate strategies under structural adjustment in Argentina Responses by industrial firms to a new set of uncertainties. New York: St. Martins Press.

Kosacoff, B. (2000b). "Business strategy under stabilization and trade openness in the 1990s". En Bernardo Kosacoff (ed.), Corporate strategies under structural adjustment in Argentina. Responses by industrial firms to a new set of uncertainties. New York: St. Martins Press.

Kuznets, S. (1955). "Economic Growth and Income Inequality". American Economic Review, 45(1): 1-28.

Levitsky, S. y Way, L. (1998). "Between a shock and a hard place: The dynamics of labor-backed adjustment in Argentina and Poland" Comparative Politics, 30(2): 171-192.

Lindenboim, J. (2007). La polémica intervención del INDEC. Indices de la discordia, Documentos individuales de miembros del Grupo Fénix (FCE - UBA) http://www.econ.uba.ar/planfenix/aportes/8/Lindenboim_La_polemica_interven cion_del_INDEC.pdf (con acceso 27.05.2011)

Majul, L. (1990). Por qué cayó Alfonsín. El nuevo terrorismo económico : los personajes, las conexiones, las claves secretas. Buenos Aires: Sudamericana.

Margheritis, A. (1999). Ajuste y reforma en Argentina (1989-1995). La economía politica de las privatizaciones, Buenos Aires: Grupo Editor Latinoamericano.

Muchnik, D. (1993). País archipiélago: las consecuencias del modelo políticoeconómico del gobierno de Menem. Buenos Aires: Planeta.

Murillo, V. (2005). Sindicatos, Coaliciones Partidarias y Reformas de Mercado en América Latina. Madrid: Siglo XXI. 
Murillo, V. (2009). Political competition, partisanship, and policy making in Latin American Public Utilities. Cambridge: Cambridge University Press.

Mussa, M. (2002). Argentina y el FMI. Del triunfo a la tragedia. Buenos Aires: Planeta.

O'Connor, J. (1973). The fiscal crisis of the State. New York: Martin's Press.

O’Donnell, G. (1988). Bureaucratic authoritarianism: Argentina, 1966-1973, in comparative perspective. Berkeley: University of California Press.

Oszlak, O. (2003). "El mito del estado mínimo: una década de reforma estatal en Argentina". Desarrollo Económico, 42(168): 519-544.

Pastor, M. y Wise, C. (1999). "Stabilization and its Discontents: Argentina's Economic Restructuring in the 1990s", World Development 27(3): 477-503.

Patterson, E. (1991). "Poverty, income inequality, and community crime rates". Criminology 29 (4): 755-776.

Rodríguez, J. (1998). Fuera de la ley: la relación entre IBM y los funcionarios públicos en los contratos informáticos del Estado. Buenos Aires: Planeta.

Scavo, C. (1996). Globalización y megatimba. Buenos Aires: Letra Buena.

Schvarzer, J. (1998). Implantación de un modelo económico. La experiencia argentina entre 1975 y el 2000. Buenos Aires: A-Z Editora.

Sevares, J. (1997). "La inserción pasiva. Costos y perspectivas". En N. Minsburg y H. Valle (eds.), El impacto de la globalización. La encrucijada económica del siglo XXI. Buenos Aires: Letra Buena.

Steger, M. y Roy, R. (2010). Neoliberalism: A very short introduction. Oxford: Oxford University Press.

Sutcliffe, B. y Glyn, A. (1999). "Still Underwhelmed: Indicators of Globalization and Their Misinterpretation". Review of Radical Political Economics 31(1):111132.

Taylor, I. (1999). Crime in Context. A Critical Criminology of Market Societies. Cambridge: Polity.

Tedesco, L. (1999). Democracy in Argentina: Hope and disillusion. Ilford: Frank Cass. 
Therborn, G. (1980). The ideology of power and the power of ideology. London: New Left Books.

Verbitsky, H. (1992). Robo para la corona: los frutos prohibidos del árbol de la corrupción. Buenos Aires: Planeta.

Waisman, C. (1990). “The Argentine Paradox". Journal of Democracy 1(1): 91-102.

Weyland, K. (2003). "Neopopulism and Neoliberalism in Latin America: how much affinity?" Third World Quarterly 24(6): 1095-1115

Wiarda, H. (1985). "Economic and political statism in Latin America". En M. Novak y M. Jackson (eds.), Latin America: Dependency or Interdependency? Washington: American Enterprise Institute for Public Policy Research. 